\title{
Was ist die soziale Dimension in der Hochschulentwicklung? Ergebnisse einer Analyse hochschulpolitischer Strategiepapiere im österreichischen Hochschulsystem
}

\author{
Michael Brandmayr \\ Institut für Soziologie, Universität Innsbruck \\ Michael.Brandmayr@uibk.ac.at
}

\section{Zusammenfassung}

Der Beitrag analysiert hochschulpolitische Strategiepapiere zur Frage, was der Begriff der sozialen Dimension im Diskurs um Hochschulpolitik in Österreich bedeutet. Es wird gefragt, welche Deutungen von Chancengerechtigkeit und Bildungsungleichheit mit ihm verbunden sind und welche Fördermaßnahmen damit begründet werden. Methodisch orientiert sich der Beitrag an der Wissenssoziologischen Diskursanalyse (Keller 20II), der Untersuchungszeitraum beträgt zehn Jahre.

Die Ergebnisse der Analyse zeigen, dass der Begriff der sozialen Dimension mit der Bologna-Deklaration erstmals im Diskurs auftritt. Darin wurde Employability zum zentralen Bildungsziel des europäischen Hochschulraums. Es zeigt sich die Deutung, durch Individualisierung, Konkurrenz und Wettbewerb die Leistungspotentiale von Individuen effizient auszuschöpfen. Der Begriff der sozialen Dimension wird in diesem Kontext nicht mehr im Sinne der Kompensation gesellschaftlicher Ungleichheit, sondern als Leistungsgerechtigkeit verstanden.

\section{Schlüsselwörter}

Hochschulpolitik, Soziale Dimension, Ungleichheitsforschung, Diskursanalyse

\section{What is the social dimension in higher education policy? Findings of an analyzes of higher education policy papers in the Austria}

\begin{abstract}
This article analyzes policy papers and is questioning the social impact on the discourse of higher education policy in Austria. It's asking for the meaning of equality and educational imparity and how it relates to supporting systems. The analysis follows the Sociology of Knowledge approach to Discourse (Keller 20II) and examines data from the last ten years. The results show that the social dimension comes along with the Bologna-Communique, in which the term employability became the objective of the European higher education system. In this documents, the interpretative scheme to use the full potential of every individual person through individualization, competition and challenge could be identified. So the social dimension doesn't mean adjustment of social inequality, but as performance linked equality.
\end{abstract}

\section{Keywords}

higher education policy, social dimension, social inequality research, discourse analyses

The author has declared that no competing interests exist. 


\section{Einleitung und Fragestellung}

Es ist ein Gemeinplatz, dass im Bildungssystem im Allgemeinen, wie auch an der Hochschule im Konkreten, Prozesse sozialer Selektion stattfinden. Umso erfreulicher, könnte man folgern, dass sich in den letzten Jahren Aktivitäten verstärken, die sich diesem Sachverhalt annehmen. Vorläufiger Höhepunkt dieser Bestrebungen ist die Veröffentlichung der "Nationale[n] Strategie zur sozialen Dimension in der Hochschulbildung" (DFI) im Jahr 2017, ein richtungsweisendes Dokument, in dem für die kommenden Jahre konkrete Zielsetzungen beschrieben und für ihre Umsetzung verantwortliche Akteure benannt wurden.

Auch das Hochschulraum-Strukturmittelprojekt „Chill die Basis: Förderung von nicht-traditionellen Studierenden" an der Universität Innsbruck widmet sich dem Fragekomplex nach sozialen und regionalen Faktoren von Bildungsungleichheit im Hochschulraum Tirol' ${ }^{1}$ Am Beginn des Projekts stand eine Bestandsaufnahme bereits umgesetzter Maßnahmen, die im Rahmen eines Workshops, durch Dokumentenanalyse von Maßnahmenbeschreibungen sowie qualitative Interviews mit Verantwortlichen der Tiroler Hochschulen durchgeführt wurde. In diesen Erhebungen wurde sichtbar, dass unter den verschiedenen AkteurInnen äußerst unterschiedliche Auffassungen existieren, welche Studierendengruppen förderbedürftig seien bzw. sich Unterstützungsmaßnahmen keinesfalls auf den Faktor der sozialen Benachteiligung beschränken sollten. Mit der Frage, woher AkteurInnen der Hochschulen Wissen und Handlungslegitimationen beziehen, rückte für das Forschungsprojekt die Ebene des hochschulpolitischen Diskurses in den Fokus.

Der folgende Beitrag stellt zentrale Ergebnisse einer Analyse von bildungspolitischen Strategiepapieren vor. Ausgangspunkt waren dabei österreichische Dokumente, aber auch internationale Dokumente aus dem Bologna-Entwicklungsprozess wurden berücksichtigt. Der Untersuchungszeitraum beträgt 20 Jahre von 1997 bis 2017. Ziel war eine inhaltliche Bestimmung des Begriffs der "sozialen Dimension“ wie eine Rekonstruktion des Diskursverlaufs. Der Beitrag versucht, zwei Fragekomplexe zu beantworten:

- Die inhaltliche Analyse von Deutungsmustern: Was bedeutet der Begriff der sozialen Dimension in der Hochschule? In welche diskursiven Formationen ist der Begriff eingebettet, und welche Deutungen über Praktiken und Maßnahmen an Universitäten werden in Zusammenhang mit dem Begriff vermittelt und legitimiert?

I An dieser Stelle möchte ich mich beim gesamten Projetteam für wertvolle Hinweise und Unterstützung dieser Forschungsarbeit bedanken.
- Die Genealogie der Aussage: Wie entsteht der Begriff? Was ist die narrative Struktur (vgl. Keller 20II) des Diskurses? Sind Aussageelemente und Deutungen aus anderen Diskursarenen nachweisbar?

Der Beitrag zeigt zunächst die theoretische Einbettung in die Diskurstheorie sowie den Stand der Forschung und stellt dann die Methodik der Untersuchung vor. Anschließend werden Ergebnisse der Diskursanalyse vorgestellt und die ermittelten inhaltlichen Deutungen des Begriffs der sozialen Dimension erläutert. Zuletzt findet sich eine Diskussion der Ergebnisse, welche abschließend die Frage der Förderung sozial benachteiligter Studierender wieder aufgreift.

\section{Theoretischer Hintergrund}

Die vorliegende Untersuchung ist in den methodologischen Kontext der Diskurstheorie bzw. in dessen Variante der Wissenssoziologischen Diskursanalyse (WDA) eingebunden. Diskurse werden im Anschluss an Keller (20II, 235) als „Komplex von Aussageereignissen und darin eingelassenen Praktiken" begriffen. Dieser weitreichende Diskursbegriff bezieht nicht nur sprachliche, sondern materialisierte Äußerungsformen und Handlungen mit ein. Die WDA setzt sich zum Ziel, die im Sprach- bzw. Symbolgebrauch konstituierten Systeme von Bedeutungen und Sinnstrukturen sozialer Realitäten zu analysieren, die besonders über die Instanz des Wissens institutionalisiert werden.

Im Sinne der Diskurstheorie wird die soziale Dimension an Hochschulen hier als Produkt einer diskursiven Konstitution gesellschaftlicher Wirklichkeit gefasst. Der Umgang mit der sozialen Dimension an Hochschulen und damit zusammenhängende Vorstellungen von Bildungsgerechtigkeit und Teilhabemöglichkeiten werden diskursiv konstituiert. Die Institutionalisierung dieses Wissens in bildungspolitischen Strategiepapieren bedeutet, dass dieses Wissens die Funktion eines objektiv existierenden, kollektiven Wissensvorrats einnimmt, auf den sich in Institutionen tätige AkteurInnen beziehen, d.h. dass das Handeln der AkteurInnen dadurch normiert und legitimiert wird. Eine Analyse der Strategiepapiere bedeutet eine Analyse der Rahmenbedingungen, innerhalb derer Fördermaßnahmen möglich werden und die damit ein spezifisches Akteurshandeln anleiten. Ebenso gibt die Analyse Auskunft über konstituierte Subjektpositionen, da diese Papiere festlegen, welche Subjekte aufgrund bestimmter Klassifikationen förderbedürftig sind und die Rahmungen erwünschten Verhaltens definieren, mit dem Nachteile idealerweise ausgeglichen werden sollten. 
Foucault (1988) betrachtet Diskurse als produktiv in dem Sinn, dass sie Wissen hervorbringen. Im Sprechen über Bildungsungleichheit wird Wissen erzeugt und in Aussageform auf verschiedenen Ebenen, d.h. als gültiges oder ungültiges, positioniert. Keller beschreibt im folgenden Zitat den Vorgang der Materialisation eines Diskurses, der auf diesen Ebenen die Gegenstände produziert, von denen er spricht. Aussagen in Strategiepapieren oder Verordnungen des EU-Rats müssen daher als Diskurseffekte erachtet werden, die den institutionellen Umgang mit der sozialen Dimension konstituieren und in den Untersuchungsfokus rücken.

„Die Welt gewinnt ihren je spezifischen Wirklichkeitscharakter für uns durch die Aussagen, die Menschen - in Auseinandersetzung mir ihr - über sie treffen, wiederholen und auf Dauer stellen. Solche Aussagen stiften nicht für die symbolischen Ordnungen und Bedeutungsstrukturen unserer Wirklichkeit, sondern sie haben auch reale Konsequenzen: Gesetze, Statistiken, Klassifikationen, Techniken, Artefakte oder Praktiken bspw. können als Diskurseffekte analysiert werden" (Keller 2OII, 237).

Diesen Aspekt genauer ausführend müssen einige Arten und Weisen, wie Wissen die soziale Wirklichkeit konstituiert, unterschieden werden: Einerseits stellt Wissen in formalisierter bzw. institutionalisierter Form die Grundlage von professionsspezifischem Handeln dar. (Bildungs-)Institutionen kanonisieren und legitimieren Wissen für bestimmte Praxisbereiche, z.B. über die Gestaltung von Curricula, und verleihen ihm damit eine Stellung in einem Wissenssystem; Zertifikate verleihen die formale Berechtigung der Anwendung des Wissens; Berufsverbände normieren dessen Anwendung und können Akteure ggf. auch ausschließen (über Dienstoder Standesrechtliche Bestimmungen). Zweitens enthält Wissen eine legitimierende Funktion nicht nur in beruflicher, sondern allgemeiner Perspektive menschlichen Handelns, die auch eine ethisch-moralische Komponente aufweist. So erzeugt Wissen darüber, was als die soziale Dimension zu verstehen ist und wie diese berücksichtigt werden soll, Legitimationen für Maßnahmen, die dadurch keiner speziellen Begründung mehr bedürfen. Schneider bezeichnet dies als „Ontologisierungstendenz" von Diskursen: „Diskurse transformieren Wissenssysteme mit ihren dazugehörenden Normierungen und Wertsystemen und verfolgen dabei in der Regel eine Ontologisierung der jeweiligen symbolischen (Wissens-) Ordnung, um ihre Kontingenz zu verdecken" (Schneider 2015, 26). Diese hat nun zwei Konsequenzen. Einmal umgesetzt, sind diese Maßnahmen in der universitären Alltagswelt normalisiert, und verwehren dadurch Einsicht in Absichten oder Strategien, die mit diesen Maßnahmen zusätzlich verfolgt wurden. Die Institutionalisierung von Prakti- ken bewirkt darüber hinaus, dass ihr zu Grunde liegende Klassifikationen und Deutungen naturalisiert werden. Maßnahmen für benachteiligte Studierende „entscheiden" demnach, wer zur Gruppe der Benachteiligten gehört, und abstrahieren, dass dies letztlich eine offene Frage darstellt, die spezifisch beantwortet wurde. Die Maßnahme selbst legitimiert also die vorherrschende Deutung.

Dies deutet einen dritten Effekt von Wissen in seiner Institutionalisierung an, der mit dem Begriff der Subjektivierung schon angesprochen wurde: Wissen wird für das Subjekt zur Grundlage von Interpretationen des Erlebten innerhalb des diskursiven Raums bzw. der Institution. Unabhängig davon, ob bestimmte Bewertungsmaßstäbe von Subjekten übernommen und Erfahrungen subjektiv in diesem Sinne gedeutet werden, sind sie da und wirken als relevante und einflussgebende Bezugsgröße. Subjektive Interpretationsschemata konstituieren sich in irgendeiner Art von Verhältnis zu dieser Bezugsgröße, es ist aber nicht möglich, sie zu ignorieren. Daher betont Keller im Anschluss an die Phänomenologie Schütz“ die „soziale Genese oder „Konstruktion“ der Deutungsschemata, die in Bewusstseinsprozessen zum Einsatz kommen und ein einfaches sinnliches "Erleben" erst in reflektierte, sinnhafte „Erfahrung“verwandeln" (Keller 2008, 78).

Um diese Vorstellungen inhaltlich zu bestimmen, wird auf das Konzept des Deutungsmusters zurückgegriffen. Der Begriff des Deutungsmusters hat Lüders/ Meuser (1997), Oevermann (2OOI), Plaß/Schetsche (200I) und anderen eine jeweils unterschiedliche Fokussierung und Konzeption erfahren. Hier soll auf die Fassung Kellers (besonders 20I4) zurückgegriffen werden, für den Deutungsmuster die Art und Weise sind, wie in Diskursen einzelne Deutungs- und Wissenselemente in Diskursen so verbunden werden, dass dadurch typische, allgemeine Erkenntnisfiguren und Zusammenhänge entstehen. Deutungsmuster sind kollektive Wissensund Interpretationsschemata, die für das Individuum Ordnung und Sinn stiften, und dabei auf anerkanntes Wissen von Diskursen zurückgreifen bzw. als deren Resultate zu verstehen sind. Deutungsmuster stellen für Keller so die Verbindung dar zwischen diskursiv generierten Schemata und dem individuellen Akt des Verstehens und Handelns. Sie sind demnach ein „Brückenkonzept', das gerade erlaubt, den Weg von der Diskursebene zur alläglichen Deutungs- und Lebenspraxis zu gehen bzw. die Verflechtungen, Adaptionen, Aneignungen und Zurückweisungen im Deutungsmustergebrauch hier und da in den Blick zu nehmen" (Keller 2014, I55).

Hinsichtlich des Standes der Forschung zu Bildungszugängen, Bildungsungleichheit und Fördermaßnahmen an den Hochschulen geben die Beiträge von Spiegler/Bednarek (20I3) sowie Müller et. al. (20I7) einen guten Überblick, da insgesamt eine große Vielfalt an Beiträgen und Forschungszugängen, und damit verbun- 
den auch Begrifflichkeiten vorliegt. Eine Fokussierung ist jene nach Chancengerechtigkeit im Hochschulzugang, hier finden sich etwa (deskriptive) Analysen zu Selektions- und Auswahlmechanismen an Hochschulen und Bildungsübergängen (Beiträge in Helsper/Krüger 2015; für Österreich Badelt et. al. 2007). Eine andere Perspektive versucht, vielfach mit Rückgriff auf Bourdieus Konzept des Habitus, eine Bestimmung von Strukturen im institutionellen Kontext der Universität, die soziale Ungleichheit reproduzieren und legitimieren (vgl. Schmitt 20IO; Kramer 20I7). In einer dritten Perspektive werden Bedingungen von Bildungsaufstiegen untersucht, meist unter dem Schlagwort der first generation students; hier lautet eine Fragestellung, warum diese in manchen Fällen gelingen und welche Faktoren Bildungsaufstiege ermöglichen (vgl. Kupfer 20I5). Während das Forschungsfeld also sehr breit ist, finden sich nur wenige Arbeiten, die mit einer ähnlichen methodologischer Rahmung arbeiten. Zu nennen wären Beiträge von Schippling (2015), die ein solches Forschungsprogramm für die französischen Elitehochschulen, die so genannten Grandes Écoles vorlegt. Für den Österreichischen Diskursraum hat Rothe (2OII) eine umfangreiche Studie vorgelegt, die sich auf den gesamten Bereich des Lebenslangen Lernens fokussiert und dort die Frage von Bildungszugängen und Chancengerechtigkeit rekonstruiert. Eine historisierende Untersuchung, mit einem dennoch aktuellen Forschungsprogramm, präsentieren Seyss-Inquart und Schmidtke $(2015,385)$, indem sie untersuchen, ,in welcher Weise Fragen der Bildungsgerechtigkeit und Chancengleichheit in Bildungsreformdiskursen in Österreich und Deutschland in den I960er und I970er Jahren" debattiert wurden. Erwähnenswert sind zudem Untersuchungen wie die österreichweit durchgeführte Studierenden-Sozialerhebung (Zaussinger et. al. 20I6), die deskriptiv die materielle Lage der Studierenden erheben. Diese empirischen Studien haben einen nicht zu vernachlässigenden Einfluss auf die hier untersuchte Frage nach der öffentlichen Deutung der „sozialen Dimension“.

Zwar zeigen sich in diesen Beiträgen Bezüge zum österreichischen Hochschuldiskurs und Forschungsperspektiven, an die hier angeknüpft werden kann; jedoch liegt (auch aufgrund der Aktualität der Fragestellung) bislang keine diskursanalytische Arbeit vor, die sich einer Analyse bildungspolitischer Strategiepapiere widmet. Diese Lücke möchte der vorliegende Beitrag schließen.

\section{Methodisches Vorgehen}

$\mathrm{Zu}$ den untersuchten Dokumenttypen zählen bildungspolitische Strategiepapiere sowie Dokumente, die im weiteren Sinne programmatische Ausrichtungskonzepte von Hochschulen darstellen und einen messbaren Einfluss auf die politische Gestaltung von Hochschu- len ausüben. Dies bedeutet, dass sie von maßgeblichen Akteuren - insbesondere Ministerien und Behörden der Hochschulverwaltung sowie Akteuren auf europäischer Ebene - verfasst wurden und über eine regulative, machtvolle Position verfügen, die ihnen Interventionen im politischen Feld der Hochschulen ermöglicht.

Ausgangspunkt der Analyse waren die aktuellsten Dokumente, wobei dem „Strategiepapier zur sozialen Dimension in der Hochschulbildung" (DFI) besondere Beachtung geschenkt wurde. Für die Analyse wurde ein zweistufiges, konstruktives und rekonstruktives Analysevorgehen gewählt. Im ersten Schritt wurden die zentralen Deutungsmuster in den aktuellsten Strategiepapieren identifiziert, und auf dieser Basis der Datenkorpus für die Untersuchung der Genealogie recherchiert und ausgewählt. Dieser zweite Schritt stellt die historisierende Analyse dar. Hier wurden 38 Dokumente aus dem Zeitraum von 1997, dem Beginn des Bologna-Prozesses ${ }^{2}$, bis heute untersucht, was eher zufällig genau einem Zeitraum von 20 Jahren entspricht. Der zeitliche Horizont sowie die Auswahl des Datenkorpus ergab sich aus dem Material und der daraus bestimmten Thesen selbst, was einem abduktiven Vorgehen entspricht.

Die analytischen Schritte (Sampling und Kodieren) orientierten sich an der grounded theory (Strauss/ Corbin I990; Strübing 20I4). Im Verfahren des offenen Kodierens wurden induktiv Kategorien ermittelt und Ausschnitte von Texten diesen Kategorien zugeordnet. Anschließend wurden ausgewählte Textteile einer Feinanalyse unterzogen, in welchem typische, d.h. immer wiederkehrende Äußerungen in ihrem Verhältnis zueinander bestimmt wurden (ähnlich wie im axialen Kodieren). Am Ende dieses Schrittes wurden Deutungsmuster aus diesen typischen Elementen rekonstruiert, wodurch eine nachvollziehbare inhaltliche Interpretation der Deutungsmuster möglich wurde. Ebenso wurden in diesem Schritt die Dimensionen des Diskurses, d.h. seine Phänomenstruktur (Keller 2008, 88) erschlossen sowie Prozesse der Formierung des Diskurses, d.h. der Verknappung und Modifikation von Aussagen.

Die folgende Darstellung fokussiert sich auf die Ergebnisse der Untersuchung, d.h. die hier beschriebenen Schritte können nicht vollumfänglich dargestellt werden. Zunächst erfolgt eine kurze historische Rekonstruktion des Diskursverlaufes, die den Entstehungskontext des Begriffs erläutert. Im Anschluss daran wird eine inhaltliche Bestimmung der Deutungsmuster der sozialen Dimension dargestellt, in der auf zwei ermittelte Deutungen genauer eingegangen wird.

2 I997 wurde das Lissabon-Abkommen unterzeichnet, welches als der Beginn des Bologna-Prozesses erachtet werden kann, da darin bereits viele grundsätzliche Absichtserklärungen formuliert sind, die auch im „eigentlichen“ Abkommen von Bologna, I999, wieder formuliert sind. 


\section{Ergebnisse der Analyse}

\subsection{Zur Rekonstruktion des Diskursverlaufes}

Die Ursprünge des Begriffes lassen sich in Life Long Learning-Strategien der Europäischen Kommission finden. Zwei bedeutende Dokumente sind die schon erwähnte Deklaration von Lissabon 1997 (DF 6) sowie die Deklaration von Bologna 1999 (DF 7). Mit der LissabonDeklaration wurde eine erste Vereinbarung geschlossen, in welcher Richtlinien zur gegenseitigen Anerkennung von Studienabschlüssen im europäischen Raum beschlossen und die Entwicklung von Systemen zur Anerkennung forciert wurden. Die Deklaration von Bologna (1999) stößt die Entwicklung eines europäischen Hochschulraums an, d.h. eine Vergleichbarkeit in der Studienarchitektur über die Einführung des ECTS-Rahmens, Zeugnisse und des Bachelor-Master Systems. Die Förderung von Mobilität unter Lehrenden und Studierenden ist ebenso ein zentrales Anliegen wie eine stärkere qualifizierende Funktion, die den Bildungssystemen Europas nun zukommen soll. Diese beiden Papiere erwähnen die soziale Dimension nicht, sind für den Diskursverlauf aber bedeutsam.

Im Prager Communiqué von 200I erscheint zum ersten Mal der Begriff der sozialen Dimension, und zwar im Kontext von Mobilität. Hier heißt es:

"Ministers reaffirmed that the objective of improving the mobility of students, teachers, researchers and administrative staff as set out in the Bologna Declaration is of the utmost importance. Therefore, they confirmed their commitment to pursue the removal of all obstacles to the free movement [...] and emphasized the social dimension of mobility." (DF 8, 2)

Die soziale Dimension bezieht sich auf die finanziellen Belastungen der erwünschten innereuropäischen Mobilität, die nicht für alle Studierende gleichermaßen tragbar sind.

Im Communiqué von Berlin 2003 (DF 9) wird der Zusammenhang von Mobilität auf „Wettbewerbsfähigkeit“ ausgedehnt und damit auf Aspekte der Studienzulassung und der ökonomischen Bedingungen während des Studiums bezogen. Das Deutungsmuster der Deklaration lautet, dass fairer Wettbewerb gleiche soziale Voraussetzungen und damit die Reduzierung von verschiedenen Formen von Benachteilgung bedürfe:

„The need to increase competitiveness must be balanced with the objective of improving the social characteristics of the European Higher Education Area, aiming at strengthening social cohesion and reducing social and gender inequalities both at national and at European level." (DF 9, I)
Dass mehr Bildungsgerechtigkeit in der Deutung der europäischen Kommission ebenso den gesellschaftlichen Zusammenhalt stärken soll, wird in den Deklarationen von Bergen 2005 (DF IO) und London 2007 (DF II) bekräftigt. Hier heißt es:

„Higher education should play a strong role in fostering social cohesion, reducing inequalities and raising the level of knowledge, skills and competences in society. Policy should therefore aim to maximise the potential of individuals in terms of their personal development and their contribution to a sustainable and democratic knowledge-based society. We share the societal aspiration that the student body entering, participating in and completing higher education at all levels should reflect the diversity of our populations." (DF II, 5)

Im Leuven/Louvain-la-Neuve Communiqué 2009 (DF I2, I-2) werden nochmals stärker die anstehenden gesellschaftlichen Herausforderungen skizziert ("challenge of an ageing population Europe, [...] globalisation and accelerated technological developments, [...] a changing labour Market [...] the consequences of a global financial and economic crisis"), und mit der Entwicklung der Universitäten verknüpft. Dazu wird fomuliert, dass die Universitäten über die Vermittlung von Kompetenzen mit diesen Herausforderungen umzugehen, über die Realisierung von Chancengerechtigkeit u.a. als ihre „dritte Mission“, d.h. gesellschaftlichen Auftrag neben Forschung und Lehre Verantwortung zu tragen hätten.

Vor diesem Hintergrund betont das Papier die Bedeutung von Life-Long-Learning und der Ausbildung von Employability. Die soziale Dimension wird noch enger an diese Parameter gebunden, indem nun auch die Praxis des Lernens an den Universitäten zum Objekt des Diskurses wird. Der neue Anspruch an Exzellenz und Qualität, der deutlich häufiger als in früheren Dokumenten genannt wird, wird als "Learning for the future“ beschrieben, das mit einer Betonung von Individualisierung, der Entwicklung von Learning Outcomes, selbstständigem und Lebenslangem Lernen verknüpft wird.

Zwischen 2009 bis 2015 , der bis dato jüngsten Konferenz in Jerewan (DF I3), und auch im aktuellsten Dokument der Europäischen Kommission 2017 (DF I4) ist die skizzierte Deutung des Begriffs innerhalb der diskursiven Formation inhaltlich stabil und nimmt eine bedeutende Position ein. Mit der Einbindung der Hochschulen in den Diskurs um das lebenslange Lernen - die zwar immer bestanden hat, sich aber seit 2009 erneut verstärkt hat - ist eine Akzentuierung der ökonomischen Deutung der sozialen Dimension feststellbar. Der Begriff erhält seine Bedeutung im europäischen Diskurs aus der Einbindung in das begriffliche Netz von Employability, Mobilität, effizientem, kompetenzorientiertem 
und lebenslangem Lernen sowie des gesellschaftlichen Zusammenhalts. Breitere Deutungsmuster in diesem Kontext sind jene des internationalen Wettbewerbs und des ökonomischen, sozialen und gesellschaftlichen Wandels. Die Aussage, dass es sich Staaten nicht mehr leisten könnte, Talente zu verlieren und ihr Humankapital nicht auszuschöpfen, ist für die Konstitution des Begriffs der sozialen Dimension zentral.

Dieser Befund gilt auch für den österreichischen Diskursverlauf zur Hochschulpolitik, in welchem der Begriff erstmals ab 2009 erscheint, aber erst ab 2013 häufiger nachzuweisen ist. Der Diskurs um Hochschulpolitik ist dabei eng mit dem Diskurs um Lebenslanges Lernen (kurz LLL) verbunden, da die Hochschulen in LLL-Strategiepapieren als ein Teil der LLL-Formation begriffen werden. So führt das erste LLL-Konsultationspapier 2008 den Bologna-Prozess im Inhaltsverzeichnis unter der Rubrik "Entwicklungen und Prozesse, denen im Rahmend der LLL: Strategie zentrale Bedeutung zukommt" (DF 5, 3) auf und erwähnt darin auch die soziale Dimension; umgekehrt nehmen hochschulpolitische Strategiepapiere diesen Aspekt auf und begreifen sich ab 2013 selbst als Teil bzw. Station eines Lebenslangen Lernens.

In der Präambel der LLL:Strategie 2020, das für das Lebenslange Lernen zentralste Dokument (DF 4), wird auf die Bedeutung von Lernen und Weiterbildung für die ökonomische und soziale Situation des Individuums und der Gesellschaft verwiesen. In aktuellen hochschulpolitischen Dokumenten nimmt dieses Deutungsmuster eine ähnlich zentrale Position ein: Dieser Aspekt wird darin so formuliert, dass es "gesellschaftliche und arbeitsmarktpolitische Ansprüche erfordern“ würden, „die sozioökonomische Zusammensetzung der Studierenden sowie Absolventinnen und Absolventen nach sozioökonomischen oder soziodemografischen und bildungsbiografischen Merkmalen an jene der Gesamtbevölkerung anzunähern". (DF 2, 30) Vor diesem Hintergrund kommt den Hochschulen stärker die Verantwortung für die "Sicherung des wirtschaftlichen Wohlstands und einer wissensbasierten Weiterentwicklung der Gesellschaft" zu, die eine „Integration möglichst breiter Gesellschaftsgruppen sowie eine optimale Nutzung und Förderung von Begabtenpotenzialen" (DF 3, 27) erfordert.

Schon Kupfer (2008) hat darauf hingewiesen, dass europäische Deutungen und Aussagen, besonders jene einer Notwendigkeit der Reform des Bildungssystems, im österreichischen Diskurs affirmativ übernommen wurden. Dieser Befund trifft auch für die vergangenen zehn Jahre des Diskursverlaufes zu. Der Begriff der sozialen Dimension kontextualisiert sich mit stellenweise wortgleichen Formulierungen vor der Aussage einer Dringlichkeit in der sozialen, gesellschaftlichen und ökonomischen Entwicklung der Gesellschaft. Besonders technische Innovationen und ein damit verbundener Qualifizierungsbedarf, wie auch verschärfte Bedingungen im ökonomischen Wettbewerb als Folge der Glo- balisierung erfordern das Aufgreifen der sozialen Dimension durch die Universitäten. Diese Entwicklungen erfordern eine Höherqualifizierung der Gesamtbevölkerung; die geistigen Kapazitäten der Studierenden, als Bildungsressourcen gedeutet, müssten quantitativ und qualitativ besser und effizienter genutzt werden.

Was bedeutet dies nun für die Gestaltung von Maßnahmen und Praktiken an den Universitäten? In den bildungspolitischen Strategiepapieren lassen sich zwei folgenreiche Implikationen nachweisen.

\subsection{Die soziale Dimension und das individuelle Leis- tungsprinzip an Universitäten}

In nationalen und europäischen Strategiepapieren wurden mit der Umgestaltung der Studienarchitektur auch Lernverhältnisse neukonzipiert. Neue Formen des Lernens setzen stärker auf Individualisierung, d.h. die Universität fokussiert sich auf die Lernumgebung, während sich Studierende Inhalte selbstständig erarbeiten und am Lernergebnis gemessen werden. Im Dokument „Dialog Hochschulpartnerschaft" wird diese Form der Hochschuldidaktik so erläutert:

"Student centred learning" orientiert sich an den Studierenden und stellt das Ergebnis des Lernprozesses in den Mittelpunkt. Der Fokus liegt auf Learning outcomes - und damit darauf, was Studierende am Ende einer Lernperiode in der Lage sind, zu tun (wie z.B. Kompetenzen, Lernergebnisse). Im Rahmen des Bologna-Prozesses liegt der Fokus in der Lehre auf Erwerb von Kompetenzen und Vermittlung von Qualifikationen. Dabei werden Kompetenzen als Ergebnisse von selbstgesteuerten, aktiven und interaktiven Lernprozessen gesehen. „Student centred learning" bedingt innovative Didaktik und stellt sich die Frage, wie gut ein Curriculum - bei Berücksichtigung des Studienaufbaus und der Gesamtbelastung - studierbar ist" (DF I5, 28).

„Learning outcomes" bedeuteten also, dass Studierende ihren Lernerfolg anhand des Ergebnisses beweisen, die erbrachte Leistung wird zum Maßstab. Die Einführung des Kompetenzbegriffs erfolgt im zitierten Dokument mit Verweis auf den Diskurs auf das Lebenslange Lernen, eine nötige Steigerung der Effizienz von Hochschulsystemen und den veränderten Bedingungen der Arbeitswelt: Die Universität sollte Kompetenzen vermitteln - etwa „selbstgesteuertes Lernen, Problemlösungskompetenz, Innovationsfähigkeit“ (DF 15, 29), die eine selbstständige, lebenslange Weiterqualifikation des Individuums gewährleisten.

Bemerkenswert ist nun, dass diese Neugestaltung auch mit der sozialen Dimension begründet wird. Dabei wird in Aussagen darauf verwiesen, dass individuali- 
sierte Lernformen größere Freiheiten (Stichwort: Anwesenheitspflicht) erlauben würden und die Drop-Out Rate von berufstätigen Studierenden oder Studierenden mit Betreuungspflichten senken würden. Student centred learning wird als inklusives Lernen verstanden, weil es sich an der persönlichen Entwicklung des Studierenden orientiert. Durch offene Lernmodalitäten und die Orientierung am Lernergebnis würde niemand im Vorfeld aufgrund von Leistungsrückständen ausgeschlossen werden.

Woher diese Leistungsrückstände kommen, wird dabei nicht thematisiert; im Begriff der Inklusion wird gerade nicht mehr zwischen verschiedenen Formen von Nachteilen unterschieden, sondern betont, dass ,jede/r anders anders" ist (Mecheril/Arens 20IO, II). Dies bedeutet, dass subjektive Folgen einer spezifischen Sozialisation (ein nicht-akademischer Habitus) einen Nachteil darstellen, die gegenüber anderen Nachteilen wie einer Lese-Rechtschreibschwäche keine spezielle Stellung einnimmt. Vielmehr werden alle Studierenden aufgrund ihrer individuellen Lebensverläufe (in denen ja Nachteile entstanden sein könnten) als potentiell förderbedürftig angenommen. Das folgende Diskursfragment zeigt dies am Beispiel der Kommunikation mit ProfessorInnen:

\section{"Angebote, wie Einführung in wissenschaftliches Arbeiten/Schreiben oder „Lernen lernen“, sind be- sonders geeignet, falls sie so gestaltet sind, dass sie von verschiedenen Gruppen nachgefragt werden. Es ist nicht selbstverständlich zu wissen, wie man mit Hochschullehrer/innen kommuniziert. Und gerade in stark nachgefragten Studien könnte ein offenes, unterstützendes Klima motivierend wirken und die Zugehörigkeit sowie Studien- und Prüfungsaktivität von Anfang an befördern." (DFI, 24)}

Bei Bourdieu (200I) wird Sprache als ein zentrales Distinktionsmerkmal genannt. Bourdieu zählt Sprechen zur inkorporierten Form sozialen Kapitals, welche durch Sozialisation gewissermaßen "vererbt" wird. Kinder aus gebildeten Elternhäusern hätten einen Startvorteil allein dadurch, dass die ihnen gewohnte Art der Sprachverwendung dem universitären Milieu gleicht. Bourdieu betont dabei, dass diese Art des Sprechens kaum nachträglich erlernt werden kann. Kinder aus bildungsnäheren Schichten verwenden habituell eine Sprechweise und Sprachcodes, die strukturell jenen der akademischen Kultur gleichen, wodurch diese Sprechweise "natürlich“ wirkt und diese Kinder als „begabter“:

„Die Professoren machen, bewusst oder unbewusst, einen Unterschied zwischen der "natürlichen" Ungezwungenheit, der Gewandtheit des Ausdrucks und eleganten Lässigkeit, und der "angestrengten"
Ungezwungenheit, die bei den Studenten aus den Volks- oder Mittelklassen häufig anzutreffen ist, und die das Bemühen verrät, sich um den Preis von Missklängen und eines »falschen « Tons den Normen des universitären Diskurses anzupassen." (Bourdieu 200I, 45)

Im Diskursfragment wird indes geäußert, dass sprachliche Fähigkeiten und Umgangsweisen erlernt werden können, und Individuen dazu angehalten werden, sich den spezifischen kulturellen und sprachlichen Regeln der Hochschule anzupassen. Es werden keine Aussagen darüber getroffen, wie und warum Bildungsungleichheit gesellschaftlich existiert, und von der strukturellen Diskriminierung innerhalb der akademischen Kultur wird nicht gesprochen. Eine erste Konsequenz der begrifflichen Formierung der sozialen Dimension besteht somit in einer Naturalisierung der Entstehungsprozesse von Bildungsungleichheit. Mit der Deutung, dass alle Formen von Nachteilen ohnehin kompensiert werden können, verliert der Begriff der sozialen Ungleichheit seine Bedeutung und die Kritik an Bildungsbenachteiligung ihre Legitimität. Dies erschwert die Einsicht in Bedingungen institutioneller Diskriminierung, auch für die betroffenen Studierenden selbst. Im Anschluss an Ernesto Laclau, kann diese Diskursstrategie als „Entleerung durch Ausweitung" (Laclau 2002, I82) interpretiert werden, denn durch die Steigerung von Förderaktivität und die Ausweitung der Bezugsgruppen können keine Prinzipien mehr erkannt werden, nach denen gefördert wird.

Die Kompensation von Nachteilen wird als Aufgabe des Einzelnen positioniert. Die Unterstützungsaufgabe der Universität besteht darin, durch das Angebot einer kompetenzorientierten, leistungsbezogenen Förderstruktur möglichst vergleichbare Bedingungen des Kompetenzerwerbs zu schaffen. Gelungene Unterstützung ist demnach solche, die einen Rahmen schafft, durch Arbeit an sich selbst bestehende Startnachteile zu kompensieren. Ziel ist ein "fairer" Wettbewerb, in dem alle Studierenden dieselben Startchancen erhalten. Hier konstituiert sich somit ein Verständnis von Chancengerechtigkeit als Leistungsgerechtigkeit, und ein zweiter Effekt der Deutung der sozialen Dimension ist demnach eine Stärkung des Leistungsprinzips durch dessen Ausweitung. Nicht-traditionelle Studierende sind demnach nicht vom Leistungsprinzip entbunden oder können im Wettbewerb ihre benachteiligte Position geltend machen, sondern gerade weil sie die Chance erhalten, sind sie aufgefordert, diese zu nutzen.

Beide Deutungen verschleiern existierende Normierungs- und Anpassungszwänge an der Universität. Während Hochschulen unter dem Schlagwort des Diversity Management eine neue Offenheit und Pluralität proklamieren und Maßnahmen gegen Diskriminierung set- 
zen, existiert innerhalb der akademischen Kultur nach wie vor eine Tendenz zur kulturellen Homogenisierung, etwa durch Sprache. Der Unterschied ist, dass der explizite Charakter des normierenden Zwangs, den Foucault für die Disziplinargesellschaft beschrieben hat, hier nicht mehr zu erkennen ist. Die Art des Zwangs ist eine andere, denn eine Anpassung an die sprachliche Norm der Hochschulen ist dem Individuum selbst überlassen. Entfaltet das hintergründig wirkende Leistungsprinzip seine Machteffekte, wird das Individuum die Anpassung an die sprachliche Norm der Hochschulen jedoch selbstverantwortlich und freiwillig vornehmen. Die Offenheit und Pluralität der akademischen Kultur ist mehr eine Scheinbare, und die Reproduktion sozialer Ungleichheit wird in ihr weiter stattfinden.

\subsection{Die soziale Dimension, die Qualität der Universi- tät und Employability}

Viele Aussagen in den Strategiepapieren vermerken eine Dynamisierung und Internationalisierung der Arbeitswelt. Um Beschäftigungsfähigkeit der AbsolventInnen zu gewährleisten wird daher die Vermittlung von Kompetenzen vorgeschlagen, die nicht nur die selbstständige Weiterqualifizierung sicherstellen (siehe 4.I); sondern, im Zusammenhang mit beruflicher Mobilität und globalen Arbeitsverhältnissen, Kompetenzen im Bereich Diversity und interkultureller Kenntnisse.

Der Zusammenhang zwischen der Qualität des Studiums, der sozialen Dimension und Employability zeigt sich demnach auf mehreren Ebenen. Einerseits, weil die soziale Dimension berücksichtigt werden muss, damit alle Kompetenzen erworben werden, die in einem qualitätsvollen Hochschulstudiums als wichtig erachtet werden. Mobilitätsprogramme sind etwa teuer; um allen Studierenden darin vermittelte Qualifikationen zu gewährleisten, muss die soziale Dimension berücksichtigt sein: "Therefore, they [die EU-MinisterInnen, Anm. M.B.] confirmed [...] to pursue the removal of all obstacles to the free movement of students, teachers, researchers and administrative staff and emphasized the social dimension of mobility." (DF 8,2)

Ein zweiter Aspekt zeigt sich darin, dass eine nichtakademische Sozialisation, ein Migrationshintergrund, Behinderung, wie aber auch andere oder gemeinsame Merkmale (Intersektionalität) als Ressource für die Hochschulen gedeutet werden. Die Nationale Strategie zur sozialen Dimension in der Hochschulbildung formuliert die "gesellschaftspolitisch relevante Dimension von Diversität [als] eine zukunftsträchtige Ressource für die Entwicklung zur Wissensgesellschaft" (DFI, 28). Der Umgang mit Diversität wird zur bedeutsamen Kompetenz für die Arbeitswelt und damit für Beschäftigungsfähigkeit erklärt; Erfahrungen mit Diversität, d.h. eine entsprechende Repräsentation nicht-traditioneller Studierender wird zum Qualitätsmerkmal für eine Hochschule.
Die Förderung nicht-traditionell Studierender steigert die Außenwahrnehmung der Institutionen, weil sie so demonstriert, dass in ihr Erfahrungen von Diversität gemacht werden können und so die Beschäftigungsfähigkeit der Studierenden und die Qualität der Ausbildung steigt. Daher ergänzt die soziale Dimension „den Exzellenzanspruch, der den Wissenschaftsbetrieb auszeichnet. Heterogenität und Diversität tragen zur weiteren Qualitätssteigerung in der Lehre bei, wovon im Endeffekt alle Studierenden und Lehrenden profitieren." (DF I, 2).

Die Deutung der sozialen Dimension als für die Universität qualitätsförderlich verdeutlicht auch die Schaffung des Diversitas-Preises im Jahr 2016. Mit diesem hat das Wissenschaftsministerium einen zusätzlichen externen Anreiz geschaffen, die soziale Dimension in der universitären Praxis zu berücksichtigen. Der Preis symbolisiert zwar, dass die Berücksichtigung der sozialen Dimension eine gesellschaftliche Bedeutung hat; jedoch erzeugt er auch Prestige und nützt den Universitäten in der Selbstpräsentation, in Rankings und der Außenwahrnehmung. Er verknüpft die soziale Dimension mit symbolischem Kapital und schafft so eine extrinsische Motivation, hinter der humanistische Motive womöglich zurückfallen. Die im vorigen Diskursfragment vermerkte Aussage, dass von Diversität „alle profitieren" (und nicht primär die Zielgruppe) unterstreicht, dass sich bildungspolitische AkteurInnen der positiven Effekte durchaus bewusst sind, die aus der spezifischen Förderung nicht-traditioneller Studierender "nebenbei ${ }^{\text {" }}$ entstehen.

\section{Diskussion}

In der Darstellung der Ergebnisse wurde deutlich, dass in den vorliegenden Strategien zur Bearbeitung der sozialen Dimension der Hochschulbildung eine wirtschaftsliberale Deutung von Bildungssystemen erkennbar ist. Die Grundannahme dieses Zugangs besteht in einer Überhöhung der Qualifikationsfunktion des Bildungssystems und der Annahme, durch Individualisierung, Konkurrenz und Wettbewerb die Leistungspotentiale von Individuen effizient und umfassend auszuschöpfen, wobei Leistung als quantifizierbarer, sichtbarer Output erachtet wird. Aus diesen Ergebnissen lassen sich zwei abschließende Thesen formulieren:

- Es wurde festgehalten, dass die soziale Dimension eine Abstraktion von ungleichheitserzeugenden Verhältnissen bedingt, sodass das konkrete Individuum seinen Erfolg bzw. sein Scheitern nicht vor universitären und gesellschaftlichen Bedingungen kontextualisiert, sondern als dafür eigenverantwortlich interpretiert. Die Orientierung an Lernergebnissen im student centered 
learning naturalisiert den Lernprozess und die dafür aufgewendete Leistung. Dies deckt sich mit dem soziologischen Befund Neckels (2010), der mit der "Kultur des Erfolgs" eine ähnliche Deutungsweise in der gegenwärtigen Ökonomie identifiziert. Neckel beschreibt darin, dass das meritokratische Prinzip, also die Beurteilung der aufgewendeten Anstrengung, in der modernen Gesellschaft immer mehr in den Hintergrund rückt. Für die Dotierung von Löhnen und Boni wird die Relation des faktisch erbrachten Aufwandes "noch nicht einmal zu Legitimationszwecken ins Feld geführt wird“. Die hier getätigte Feststellung einer Ausweitung des Leistungsprinzips an der Universität muss also so verstanden werden, dass sie in der Subjektivierungsform der Studierenden die Bedeutung konkreter Ergebnisse erhöht und diese einfordert. Es kann also angenommen werden, dass die faktische Leistungsanstrengung, d.h. die aufgewendete Zeit und Mühe steigt, die Studierende aufbringen, um Employability zu erwerben (und zwar nicht nur an der Universität, sondern auch in Form von Praktika, Volontariaten, Auslandsaufenthalten usw.). Die Abstraktion von ungleichheitserzeugenden Verhältnissen bedeutet zudem, dass die Effekte benachteiligender Strukturen verfremdet werden - schafft es ein Studierender, diesen zu trotzen, und ein anderer nicht, ist dieser "selbst schuld“ - denn der andere Studierende "beweist", dass die Leistung dennoch möglich gewesen wäre. Dies untermauern auch neuere Befunde in der Befragung von BildungsaufsteigerInnen, die, rückblickend auf ihr Studium, keine sie diskriminierenden Studienbedingungen ausmachen können (vgl. Gerhartz-Reiter 2016)

Ein Universitätsabschluss wird nicht mehr als abschließendes, endgültiges Erreichen von Bildungszielen verstanden, und es besteht keine Gewissheit darüber, ob mit der eingesetzten Lernleistung das Ziel einer beruflichen Anstellung erworben werden kann. Denn ob Employability erreicht wurde, bestimmt sich in $\mathrm{Ab}$ hängigkeit vermuteter Jobchancen und der Performance des Studierenden in Konkurrenz zu seinen MitbewerberInnen am Arbeitsmarkt und deren erworbener Qualifikationen. Aus diesen Befunden können Hypothesen für Prozesse der Subjektkonstitution an der Universität getroffen werden, weil nicht nur der Leistungsdruck, sondern auch die Konkurrenzsituation sich verschärft darstellt. Die angebotene Subjektposition zeichnet sich durch ihre Unklarheit aus, sie entspricht einer Leerstelle, deren zentrale
Elemente Unbeständigkeit und Unbestimmtheit darstellen. Sie nimmt als zentralen Richtwert für gelungene Subjektivität den Markt als Richtwert, die eigene Performance ergibt sich aus dem Abschneiden gegenüber der Konkurrenz. Die Subjektivierungsform, die gegenwärtig an den Universitäten vermittelt wird, entspricht der eines „Wettbewerbsindividualismus" (Dörre 2014, 395). Die Position eines Studierenden konstituiert sich (scheinbar oder tatsächlich) entsprechend seines Erfolges, der - zu Recht oder nicht - in direkten Zusammenhang zu dessen Leistung gesetzt wird. Durch die Leerstelle ist eine temporäre Subjektposition dabei immer bedroht, was Studierende zu kontinuierlicher Arbeit an sich selbst zwingt. Wie Dörre hinweist, führt dieses Gefühl von Konkurrenz zu einer Fragmentierung des Klassenbewusstseins. Der Begriff der sozialen Klasse scheint im Diskurs zu verschwinden und kann nicht mehr als Bezugspunkt für kollektive Interessen gelten.

\section{Fazit}

Der Beitrag untersuchte, wie in bildungspolitischen Strategiepapieren über die "soziale Dimension“ in der Hochschule gesprochen wird und wie sich die Deutung des Begriffs historisch konstituiert hat. Dabei wurde vermerkt, dass der Begriff eng an weitere Ziele wie Employability, Mobilität und weitere gesellschaftliche Ziele gebunden und von diesen in seiner Bedeutung determiniert ist. Hintergründig befördert er so das Leistungsprinzip an den Universitäten und verschleiert gesellschaftliche Entstehungsprozesse sozialer Ungleichheit, während die Einlösung individueller Chancen als Problem des Einzelnen formuliert wird. Bildungspolitische Strategiepapiere verzichten auf den Begriff der Bildungsungleichheit und klammern dessen gesellschaftlichen Entstehungsprozess aus. Es finden sich keine zielgruppenspezifischen Vorschläge, sondern es wird eine Förderstruktur vorgeschlagen, die Bildungsungleichheit als individuell zu bearbeitendes Problem positioniert.

Aus einer diskurstheoretischen Perspektive wird für eine Rückkehr zum Begriff der Bildungsungleichheit plädiert, um ungleichheitsfördernde Strukturen problematisieren zu können. Gleichzeitig möchte der Beitrag auf die oben beschriebenen Konsequenzen jüngster Universitätsreformen aufmerksam machen, die weitgehend unbemerkt von der Öffentlichkeit unternommen wurden, und über die sich stillschweigend eine neue Leistungskultur an den Universitäten etablieren kann. Zu beiden Aspekten wäre eine breitere öffentliche Debatte wünschenswert. Ebenso bedarf es einer intensiveren Debatte wie umfassend universitäre Fördermaßnah- 
men entwickelt und bereitgestellt werden sollen, um die soziale Selektivität im tertiären Bereich abzumildern. Auch dazu arbeitet das HRSM-Projekt "Chill die Basis“ an der Universität Innsbruck, in dessen Rahmen diese Forschungsarbeit entstanden ist, und wird dazu bald Erkenntnisse liefern (für eine erste Einschätzung dazu siehe Brandmayr et. al. 20I8).

\section{Literatur}

Althusser, Louis (2010), Ideologie und ideologische Staatsapparate, in Ders. (Hg.), Gesammelte Schriften, I. Halbband, Hamburg: VSA.

Brandmayr, Michael/Bernadette Müller-Kmet/Ina Hanselmann (2018), Maßnahmen zur Ermöglichung von Bildungsaufstieg zwischen bildungspolitischen Vorgaben, sozialwissenschaftlicher Forschung und institutioneller Praxis, in: Magazin Erwachsenenbildung, Vol. 2/2018, 90-98.

Badelt, Christoph/Wolfhard Wegscheider/Heribert Wulz (2007), Hochschulzugang in Österreich, Graz: Leykam.

Baader, Meike/Tatjana Freytag (Hg.) (2017), Bildung und Ungleichheit in Deutschland, Wiesbaden: Springer.

Behrmann, Laura/Falk Eckert/Andreas Gefken /Peter A. Berger (Hg.) (2018), ,Doing Inequality': Prozesse sozialer Ungleichheit im Blick qualitativer Sozialforschung, Wiesbaden: Springer.

Bourdieu, Pierre (200I), Die konservative Schule, in: Ders. (Hg.), Wie die Kultur zum Bauern kommt, Hamburg: VSA, 25-52.

Bruneforth, Michael/ Lorenz Lassnigg, Stefan Vogtenhuber, Claudia Schreiner, Simone Breit (Hg.) (2016), Nationaler Bildungsbericht Österreich 2015, Band I: Das Schulsystem im Spiegel von Daten und Indikatoren, Graz: Leykam.

Dörre, Klaus (20I4), Fragmentiertes Klassenbewusstsein? Zur subjektiven Dimension kapitalistischer Landnahmen, in: Bauer, Ulrich/ Axel Bolder/Helmut Bremer/Rolf Dobischat/ Günter Kutscha (Hg.), Expansive Bildungspolitik-Expansive Bildung? Wiesbaden: Springer, 377-399.

Foucault, Michel (1973/1988), Archäologie des Wissens. 3. Aufl, Frankfurt am Main: Suhrkamp.

Gerhartz-Reiter, Sabine (2016), Erklärungsmuster für Bildungsaufstieg und Bildungsausstieg: wie Bildungskarrieren gelingen, Wiesbaden: Springer.

Helsper, Werner/Heinz-Hermann Krüger (Hg.), Auswahl der Bildungsklientel: Studien zur Schul- und Bildungsforschung, Vol 55, Wiesbaden: Springer.

Keller, Reiner (2014), Wissenssoziologische Diskursforschung und Deutungsmusteranalyse, in: Behnke, Cornelia/Diana Lengersdorf/ Sabine Scholz (Hg.), Wissen
- Methode - Geschlecht, Wiesbaden: Springer, I43I59.

Keller, Reiner (20II), Wissenssoziologische Diskursanalyse: Grundlegung eines Forschungsprogramms, Wiesbaden: Springer.

Keller, Reiner (2008), Diskurse und Dispositive analysieren: Die wissenssoziologische Diskursanalyse als Beitrag zu einer wissensanalytischen Profilierung der Diskursforschung, in: Historical Social Research, Vol. 33/I, 73-107.

Kramer, Rolf-Torsten (2017), Habitus "und „kulturelle Passung", in: Rieger-Ladich, Markus/Christian Grabau, Pierre Bourdieu (Hg.), Pädagogische Lektüren, Wiesbaden: Springer, I83-205.

Kupfer, Antonia (2008), Diminished states? National Power in European Education Policy, in: British Journal of Educational Studies, Vol. 56 (3), 286-303.

Kupfer, Antonia (2015), Educational Upward Mobility: Practices of Social Changes, Ort: Palgrave Macmillan UK.

Laclau, Ernesto (2002), Tod und Wiederauferstehung der Ideologietheorie, in: Ders. (Hg.), Emanzipation und Differenz, Wien: Turia+ Kant, 174-200.

Lüders, Christian/ Michael Meuser (1997), Deutungsmusteranalyse, in: Hitzler Roland/Anne Honer (Hg.), Sozialwissenschaftliche Hermeneutik: Eine Einführung, Opladen: Barbara Budrich, 57-8I.

Mecheril Paul/Susanne Ahrens (2010), Schule - Vielfalt - Gerechtigkeit. Schlaglichter auf ein Spannungsverhältnis, das die politische und erziehungswissenschaftliche Diskussion in Bewegung gebracht hat, in: Lernende Schule, Vol. 49/2010, 9-II.

Müller, Walter/Reinhard Pollak/David Reimer/Steffen Schindler (2017), Hochschulbildung und soziale Ungleichheit, in: Becker, Rolf (Hg). Lehrbuch der Bildungssoziologie, Wiesbaden: Springer, 309-358.

Neckel, Sighard (2010), Refeudalisierung der Ökonomie: Zum Strukturwandel kapitalistischer Wirtschaft, MPIfG working paper, No. Io/6, Internet: http:// www.mpifg.de/pu/workpap/wpıo-6.pdf (Zugriff: 29.0I.2018).

Oevermann, Ulrich (200I), Die Struktur sozialer Deutungsmuster-Versuch einer Aktualisierung, in: Sozialer Sinn. Zeitschrift für hermeneutische Sozialforschung, Vol. Nr. (I), 35-82.

Plaß, Christine/Michael Schetsche (200I), Grundzüge einer wissenssoziologischen Theorie sozialer Deutungsmuster, in: Sozialer Sinn. Zeitschrift für hermeneutische Sozialforschung, Vol. Nr. (3), 511-536.

Rothe, Daniela (20II), Lebenslanges Lernen als Programm. Eine diskursive Formation in der Erwachsenenbildung, Frankfurt/M.: Campus.

Schippling, Anne (2013), Am Übergang in eine französische Elitehochschule. Diskursanalytische Perspek- 
tiven, in: Siebholz, Susanne/Edina Schneider/Susann Busse/Sabine Sandring/Anne Schippling (Hg.), Prozesse sozialer Ungleichheit, Wiesbaden: Springer, 217-227.

Schmidtke, Adrian/Julia Seyss-Inquart (2015), Bildungspolitik und Bildungsreform, in: Susann Fegter/Fabian Kessl/Antje Langer/Marion Ott/Daniela Rothe/Daniel Wrana (Hg.), Erziehungswissenschaftliche Diskursforschung, Wiesbaden: Springer, 385-403.

Schmitt, Lars (2010), Bestellt und nicht abgeholt: Soziale Ungleichheit und Habitus-Struktur-Konflikte im Studium, Wiesbaden: Springer.

Schneider, Werner (2015), Dispositive...-überall (und nirgendwo)? Anmerkungen zur Theorie und methodischen Praxis der Dispositivforschung, in: Othmer, Julius/Andreas Weich (Hg.), Medien-Bildung-Dispositive, Wiesbaden: Springer, 2I-4O.

Spiegler, Thomas/Antje Bednarek (20I3), First-Generation Students: What we ask, what we know and what it means: An international review of the state of research, in: International Studies in Sociology of Education, Vol. 23(4), 318-337.

Strauss, Amseln/Juliet Corbin (1990), Grounded Theory Research: Procedures, Canons and Evaluative Criteria, in: Zeitschrift für Soziologie, Vol. I9 (6), 4I8-427.

Strübing, Jörg (2014), Grounded Theory. Zur sozialtheoretischen und epistemologischen Fundierung eines pragmatistischen Forschungsstils, Wiesbaden: Springer.

Zaussinger, Sussane/ Martin Unger et al. (2016), Studierenden-Sozialerhebung 2015. Bericht zur sozialen Lage der Studierenden. Band I. Hochschulzugang und StudienanfängerInnen, Institut für höhere Studien (IHS), Internet: http://irihs.ihs.ac.at/3978/I/Studierenden_Sozialerhebung_2OI5_BandI_AnfaengerInnen.pdf (Zugriff: 29.06.2018).

\section{Autor}

Michael Brandmayr hat an der Universität Innsbruck Erziehungswissenschaft studiert und 2017 mit einer diskursanalytischen Arbeit zu jüngsten Reformen im österreichischen Bildungssystem und der Frage, wie sich dadurch ein neues Leitbild von idealem Lernen konstitutiert hat, promoviert. Von 2012 bis 2016 war er am Institut für LehrerInnenbildung als Senior Lecturer tätig, von 2017 bis Juli 2018 als Post-Doc Mitarbeiter am Institut für Soziologie im Projekt „Chill die Basis: Förderung von nicht-traditionellen Studierenden“. Parallel dazu führte er am Institut für Erziehungswissenschaften ein Projekt zur Analyse der politischen Bildung in Tirol durch. Gegenwärtig ist er externer Lehrbeauftragter an den Universitäten Wien, Bozen und Innsbruck. 


\section{Quellenverzeichnis}

\begin{tabular}{|c|c|c|c|c|}
\hline Nr. & Titel/Bezeichnung & $\begin{array}{l}\text { Dokumententyp und } \\
\text { Autorlnnen }\end{array}$ & $\begin{array}{l}\text { Erschein- } \\
\text { ungsjahr }\end{array}$ & Quelle \\
\hline DF 1 & $\begin{array}{l}\text { Nationale Strategie zur } \\
\text { sozialen Dimension in } \\
\text { der Hochschulbildung }\end{array}$ & $\begin{array}{l}\text { Bundesministerium für } \\
\text { Wissenschaft, Forschung und } \\
\text { Wirtschaft }\end{array}$ & 2017 & $\begin{array}{l}\text { Online-Dokument, https://www.bmwfw. } \\
\text { gv.at/Presse/AktuellePresseMeldungen/ } \\
\text { Documents/2017_Strategien_Book_WEB\%20 } \\
\text { nicht\%20barrierefrei.pdf } \\
\text { [Zugriff am 14.10.2017] }\end{array}$ \\
\hline DF 2 & $\begin{array}{l}\text { Der gesamt- } \\
\text { österreichische } \\
\text { Universitäts- } \\
\text { entwicklungsplan } \\
2016-2021\end{array}$ & $\begin{array}{l}\text { Bundesministerium für } \\
\text { Wissenschaft, Forschung und } \\
\text { Wirtschaft }\end{array}$ & 2015 & $\begin{array}{l}\text { Online-Dokument, https://wissenschaft. } \\
\text { bmwfw.gv.at/fileadmin/user_upload/ } \\
\text { wissenschaft/publikationen/2015_goe_UEP- } \\
\text { Lang.pdf }\end{array}$ \\
\hline DF 3 & $\begin{array}{l}\text { Österreichischer } \\
\text { Hochschulplan. Der } \\
\text { Gestaltungsprozess } \\
\text { zur Weiterentwicklung } \\
\text { des österreichischen } \\
\text { Hochschulraums }\end{array}$ & $\begin{array}{l}\text { Bundesministerium für } \\
\text { Wissenschaft und Forschung }\end{array}$ & 2010 & $\begin{array}{l}\text { Online-Dokument, http:// hochschulplan. } \\
\text { at/wp-content/uploads/2012/12/ } \\
\text { Hochschulplan.pdf [Zugriff am 14.10.2017] }\end{array}$ \\
\hline DF 4 & $\begin{array}{l}\text { LLL 2020: Strategie zum } \\
\text { lebensbegleitenden } \\
\text { Lernen in Österreich }\end{array}$ & $\begin{array}{l}\text { Bundesministerium für } \\
\text { Unterricht, Kunst und } \\
\text { Kultur; } \\
\text { Bundesministerium } \\
\text { für Wissenschaft } \\
\text { und Forschung; } \\
\text { Bundesministerium für } \\
\text { Arbeit, Soziales und } \\
\text { Konsumentenschutz; } \\
\text { Bundesministerium für } \\
\text { Wirtschaft, } \\
\text { Familie und Jugend }\end{array}$ & 2011 & $\begin{array}{l}\text { Online-Dokument, https:// } \\
\text { erwachsenenbildung.at/themen/ } \\
\text { lebenslanges_lernen/oesterreichische_ } \\
\text { strategie/aktuell.php [Zugriff am 14.10.2017] }\end{array}$ \\
\hline DF 5 & $\begin{array}{l}\text { Wissen - Chancen - } \\
\text { Kompetenzen. Strategie } \\
\text { zur Umsetzung des } \\
\text { lebenslangen Lernens } \\
\text { in Österreich }\end{array}$ & BMUKK & 2008 & $\begin{array}{l}\text { Online-Dokument, https:// } \\
\text { erwachsenenbildung.at/downloads/themen/ } \\
\text { LLL2008_Konsultationspapier_03c.pdf } \\
\text { [Zugriff am 14.10.2017] }\end{array}$ \\
\hline DF 6 & Lissabon Communiqué & $\begin{array}{l}\text { Ministers of the } \\
\text { European Higher } \\
\text { Education Area (EHEA) }\end{array}$ & 1997 & $\begin{array}{l}\text { Online-Dokument, https://rm.coe. } \\
\text { int/168007f2e5 [Zugriff am 14.10.2017] }\end{array}$ \\
\hline DF 7 & Bologna Declaration & $\begin{array}{l}\text { Ministers of the } \\
\text { European Higher } \\
\text { Education Area (EHEA) }\end{array}$ & 1999 & $\begin{array}{l}\text { Online-Dokument, https:// www.eurashe. } \\
\text { eu/library/bologna_1999_bologna- } \\
\text { declaration-pdf/ [Zugriff am 14.10.2017] }\end{array}$ \\
\hline DF 8 & Prag Communiqué & $\begin{array}{l}\text { Ministers of the } \\
\text { European Higher } \\
\text { Education Area (EHEA) }\end{array}$ & 2001 & $\begin{array}{l}\text { Online-Dokument, http://media.ehea. } \\
\text { info/file/2001_Prague/44/2/2001_ } \\
\text { Prague_Communique_English_553442.pdf } \\
\text { [Zugriff am 14.10.2017] }\end{array}$ \\
\hline DF 9 & Berlin Communiqueì & $\begin{array}{l}\text { Ministers of the } \\
\text { European Higher } \\
\text { Education Area (EHEA) }\end{array}$ & 2003 & $\begin{array}{l}\text { Online-Dokument, http://media.ehea. } \\
\text { info/file/2003_Berlin/28/4/2003_Berlin_ } \\
\text { Communique_English_577284.pdf [Zugriff } \\
\text { am 14.10.2017] }\end{array}$ \\
\hline
\end{tabular}




\begin{tabular}{|c|c|c|c|c|}
\hline Nr. & Titel/Bezeichnung & $\begin{array}{l}\text { Dokumententyp und } \\
\text { Autorlnnen }\end{array}$ & $\begin{array}{l}\text { Erschein- } \\
\text { ungsjahr }\end{array}$ & Quelle \\
\hline $\begin{array}{l}\text { DF } \\
10\end{array}$ & Bergen Communiqueì & $\begin{array}{l}\text { Ministers of the } \\
\text { European Higher Education } \\
\text { Area (EHEA) }\end{array}$ & 2005 & $\begin{array}{l}\text { Online-Dokument, http://media.ehea. } \\
\text { info/file/2005_Bergen/52/0/2005_Bergen__ } \\
\text { Communique_english_580520.pdf [Zugriff am } \\
\text { 14.10.2017] }\end{array}$ \\
\hline $\begin{array}{l}\text { DF } \\
11\end{array}$ & $\begin{array}{l}\text { London Communiqué } \\
\text { Towards the European } \\
\text { Higher Education Area: } \\
\text { responding to } \\
\text { challenges in a } \\
\text { globalised world }\end{array}$ & $\begin{array}{l}\text { Ministers of the } \\
\text { European Higher Education } \\
\text { Area (EHEA) }\end{array}$ & 2007 & $\begin{array}{l}\text { Online-Dokument, https://www.eqar.eu/ } \\
\text { fileadmin/documents/bologna/London- } \\
\text { Communique-18May2007.pdf [Zugriff am } \\
\text { 14.10.2017] }\end{array}$ \\
\hline $\begin{array}{l}\text { DF } \\
12\end{array}$ & $\begin{array}{l}\text { Leuven and Louvain-la- } \\
\text { Neuve }\end{array}$ & $\begin{array}{l}\text { Ministers of the } \\
\text { European Higher Education } \\
\text { Area (EHEA) }\end{array}$ & 2009 & $\begin{array}{l}\text { Online-Dokument, http://media.ehea.info/ } \\
\text { file/2009_Leuven_Louvain-la-Neuve/06/1/ } \\
\text { Leuven_Louvain-la-Neuve_Communique_ } \\
\text { April_2009_595061.pdf [Zugriff am 14.10.2017] }\end{array}$ \\
\hline $\begin{array}{l}\text { DF } \\
13\end{array}$ & $\begin{array}{l}\text { Yerevan Bologna } \\
\text { Communiqué }\end{array}$ & $\begin{array}{l}\text { Ministers of the } \\
\text { European Higher } \\
\text { Education Area (EHEA) }\end{array}$ & 2015 & $\begin{array}{l}\text { Online-Dokument, http:// bologna- } \\
\text { yerevan2015.ehea.info/files/ } \\
\text { YerevanCommuniqueFinal.pdf [Zugriff am } \\
\text { 14.10.2017] }\end{array}$ \\
\hline $\begin{array}{l}\text { DF } \\
14\end{array}$ & $\begin{array}{l}\text { Eine europäische } \\
\text { Erneuerungsagenda für } \\
\text { die Hochschulbildung }\end{array}$ & Europäische Kommission & 2017 & $\begin{array}{l}\text { Online-Dokument, http://ec.europa.eu/ } \\
\text { transparency/regdoc/rep/1/2017/DE/COM- } \\
\text { 2017-247-F1-DE-MAIN-PART-1.PDF }\end{array}$ \\
\hline $\begin{array}{l}\text { DF } \\
15\end{array}$ & $\begin{array}{l}\text { Dialog } \\
\text { Hochschulpartner- } \\
\text { schaft. } \\
\text { Empfehlungen zur } \\
\text { Zukunft } \\
\text { des tertiären Sektors }\end{array}$ & $\begin{array}{l}\text { Bundesministerium } \\
\text { für Wissenschaft und } \\
\text { Forschung }\end{array}$ & 2010 & $\begin{array}{l}\text { Online-Dokument, http:// } \\
\text { hochschulplan.at/wp-content/ } \\
\text { uploads/2012/06/Endbericht_Dialog_ } \\
\text { Hochschulpartnerschaft-2010.pdf [Zugriff } \\
\text { am 14.10.2017] }\end{array}$ \\
\hline
\end{tabular}


